\title{
SAR Compliance Assessment of PMR 446 and FRS Walkie-Talkies
}

\author{
Günter Vermeeren, ${ }^{*}$ Wout Joseph, and Luc Martens \\ Department of Information Technology, iMinds/UGent, Ghent, Belgium
}

\begin{abstract}
The vast amount of studies on radiofrequency dosimetry deal with exposure due to mobile devices and base station antennas for cellular communication systems. This study investigates compliance of walkie-talkies to exposure guidelines established by the International Commission on NonIonizing Radiation Protection and the Federal Communications Committee. The generic walkietalkie consisted of a helical antenna and a ground plane and was derived by reverse engineering of a commercial walkie-talkie. Measured and simulated values of antenna characteristics and electromagnetic near fields of the generic walkie-talkie were within $2 \%$ and $8 \%$, respectively. We also validated normalized electromagnetic near fields of the generic walkie-talkie against a commercial device and observed a very good agreement (deviation $<6 \%$ ). We showed that peak localized specific absorption rate (SAR) induced in the oval flat phantom by the generic walkietalkie is in agreement with four commercial devices if input power of the generic walkie-talkie is rescaled based on magnetic near field. Finally, we found that SAR of commercial devices is within current SAR limits for general public exposure for a worst-case duty cycle of $100 \%$, that is, about 3 times and 6 times lower than the limit on the $1 \mathrm{~g}$ SAR $(1.6 \mathrm{~W} / \mathrm{kg})$ and $10 \mathrm{~g}$ SAR $(2 \mathrm{~W} / \mathrm{kg})$, respectively. But, an effective radiated power as specified by the Private Mobile Radio at $446 \mathrm{MHz}$ (PMR 446) radio standard can cause localized SAR exceeding SAR limits for $1 \mathrm{~g}$ of tissue. Bioelectromagnetics. 36:517-526, 2015. C 2015 Wiley Periodicals, Inc.
\end{abstract}

Key words: modeling; exposure; two-way radio transceiver; absorption assessment; helical antenna

\section{INTRODUCTION}

Although a vast amount of studies on dosimetry deal with exposure due to mobile devices and base station antennas for cellular communication systems, the mobile phone is not the only device operated in proximity of the human head. Using the case study of the walkie-talkie, we draw attention to a device that gained interest as an alternative to cellular phones for short-range communications. Walkie-talkies, or twoway radios, operate close to the human head as is the case for mobile phones. Typical operating positions of a walkie-talkie are in front of the face, whereas a mobile phone is typically operated next to the ear. When operating a walkie-talkie, its antenna could be just in front of the eye. The International Commission on Non-Ionizing Radiation Protection (ICNIRP) restricts local-averaged specific absorption rate (SAR) in $10 \mathrm{~g}$ (applicable in Europe) based on cataract in the eye of a rabbit [ICNIRP, 1998]. As opposed to mobile phones, no or little attention is paid to walkie-talkies in dosimetry, although compliance tests also apply to these devices. Cecil et al. [2014] and Dimbylow et al. [2003] investigated numerically peak $10 \mathrm{~g}$ localized SAR induced by Terrestrial Trunked Radio (TETRA) transmitters positioned around the human body.
In recent years, walkie-talkies are more frequently encountered as a short-range communication device (e.g., adults give a walkie-talkie to their children when they go to play outside their house; parking lot attendants employ a walkie-talkie to direct the traffic inside a parking lot to assist drivers in finding a free parking spot, etc.) Advantages of walkie-talkies with respect to cellular phones are their ease-of-use (push-to-talk), their inexpensiveness, and free-of-charge communications.

In Europe, a walkie-talkie operates according to the Private Mobile Radio at $446 \mathrm{MHz}$ (PMR 446) standard; in the United States, Family Radio Service (FRS) is authorized for license-free short-range voice communication [ERC, 1998; ETSI, 2001a, 2015;

Conflicts of interest: None.

*Correspondence to: Günter Vermeeren, iMinds/UGent-INTEC, Gaston Crommenlaan 8 box 201, Ghent B-9050, Belgium.

E-mail: gunter.vermeeren@intec.ugent.be

Received for review 21 August 2014; Accepted 13 August 2015

DOI: $10.1002 /$ bem. 21935

Published online 6 September 2015 in Wiley Online Library (wileyonlinelibrary.com). 
FCC, 2015]. FRS uses 14 channels within frequency bands $462.5625-462.7125 \mathrm{MHz}$ and $467.5625-$ 467.7125 MHz. PMR 446 specifies eight channels within frequency range 446.0-446.1 MHz. Each channel has a bandwidth of 25 and $12.5 \mathrm{kHz}$ for FRS and PMR 446, respectively. Maximum allowed Effective Radiated Power (ERP) is $500 \mathrm{~mW}$ for both systems. Frequency modulation (FM) has been adopted as modulation scheme. Relative low frequency, effective radiated power of $500 \mathrm{~mW}$, use of walkie-talkies by children, position in front of the face, and limited number of exposure studies for walkie-talkies attracted our attention to these devices.

The objective of this study was to develop and evaluate an accurate helical antenna of a generic walkie-talkie for compliance assessment with ICNIRP [1998] and FCC [2001] guidelines. We designed a generic walkie-talkie and evaluated it in free space and below a flat phantom against a real device. This validation between model and real device will allow us to use the walkie-talkie model in future studies (e.g., to evaluate absorption in anatomical human body models.) To our knowledge, compliance of walkie-talkies operating according to PMR 446 and FRS standards under worst-case conditions has not yet been investigated in literature. In addition, compliance of four commercial walkie-talkies was evaluated. The model is obtained by reverse engineering and is validated by reflection, near-field, and far-field measurements. The use of helical monopole antennas as a model for portable handheld devices have been mainly discussed for mobile-phone technologies [Lazzi and Gandhi, 1998; Koulouridis and Nikita, 2004] operating in frequency bands around $900 \mathrm{MHz}$ and $1800 \mathrm{MHz}$. In this study, a helical monopole antenna model of a walkie-talkie operating at $446 \mathrm{MHz}$ was designed to study electromagnetic fields induced in the human head by walkie-talkies. A model is preferred over a real walkie-talkie device mainly for two reasons: firstly, antenna characteristics (e.g., reflection at input terminals of a model can be measured accurately); secondly, input power is easily adjustable as one can feed using a signal generator contrary to a real device. The model also allows study of influence of dimensions and material parameters on antenna characteristics and absorption or SAR assessment in realistic human head models.

\section{MATERIALS AND METHODS}

\section{Generic Walkie-Talkie Design}

We developed a generic walkie-talkie by reverse engineering of a commercially available PMR 446 radio, that is, the COBRA MT500 (Cobra Electronics, Chicago, IL) shown in Figure 1a. We dismantled the COBRA MT500 and developed a generic walkietalkie (Fig. 1b) based on physical dimensions of the antenna structure (including coating around antenna) and Printed Circuit Board (PCB). The model consisted of a helical antenna mounted on a rectangular ground plane. Terminals of the source connected the helical antenna and ground plane of the model. To limit complexity of the model, the PCB of the walkie-talkie was replaced by a perfectly conducting ground plane and the case was removed. The helical antenna fits in a dielectric cover. This cover around the helical antenna influences antenna characteristics of the model. Dielectric properties of this cover were unknown and we derived properties by tuning simulated antenna characteristics and near-field distributions to measured results.

\section{SAR Assessment}

We tested SAR compliance for the generic walkie-talkie as well as for four commercially available walkie-talkies (Table 1) according to IEC standard 62209-2 [IEC, 2005]. We placed the walkie-talkie at a distance (d) below an oval flat phantom as shown in Figure 2. We did not take the user's hand into account. IEC standard 62209-2 [IEC, 2005] for compliance testing does not specify hand position because dosimetric studies [Balzano et al., 1995; Kuster et al., 1997a, b; Meyer et al., 2001] suggest that excluding the hand in modelling constitutes a conservative case scenario for SAR in head.

\section{Measurement Setup}

Measurements of reflection and input impedance were performed in an anechoic room using a vector network analyzer (VNA) (type HP8710, Agilent Technologies [formerly Hewlett Packard], Palo Alto, CA). The far-field characteristics in terms of Total Radiated Power (TRP) and the Effective Radiated Power (ERP) were measured. TRP measurements were performed in a reverberation chamber according to TCO'01 Certification of Mobile Phones [TCO Development, 2008]. Measurement uncertainty was $1 \mathrm{~dB}$. ERP measurement was performed in an anechoic room according to European Telecommunications Standards Institute (ETSI) standard EN 300 296-2:2001-03 [ETSI, 2001b]. Measurement uncertainty was $3.3 \mathrm{~dB}$, which is within the uncertainty boundary of $6 \mathrm{~dB}$ specified by the ETSI standard. This large uncertainty is due to simplifications built into measurement methodology to reduce time and costs of tests. For walkie-talkies, ERP was only measured in eight different directions in the azimuth plane 


\section{(a)}

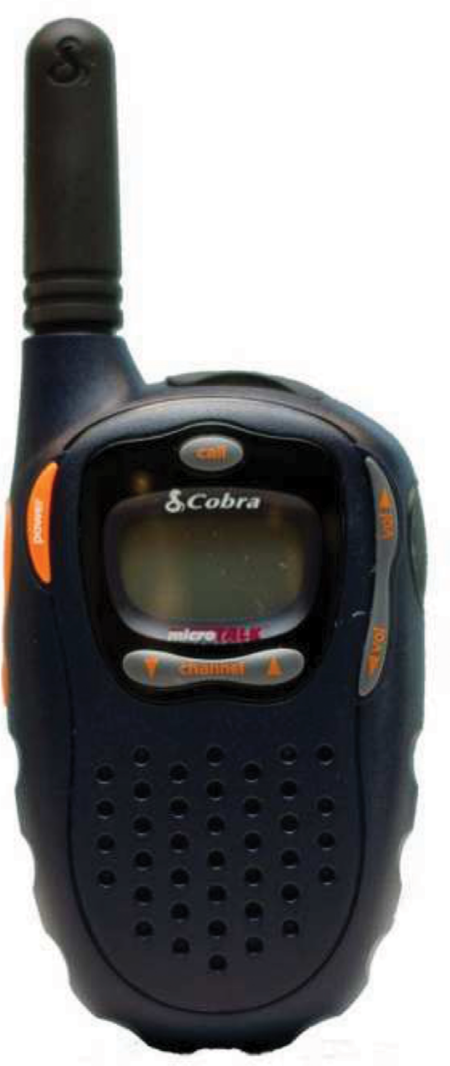

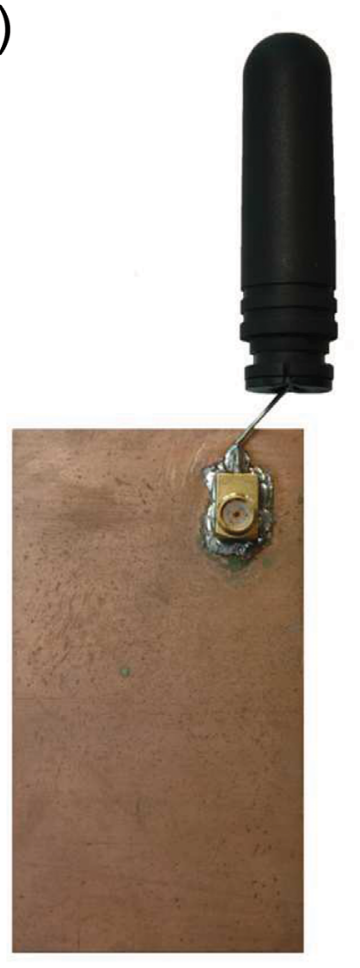

Fig. 1. (a) COBRA MT500 (Cobra Electronics); (b) back of derived model with helical antenna enclosed by a dielectric cover (bazooka balun is not shown).

resulting in an uncertainty of $3.3 \mathrm{~dB}$. Near fields were measured in an indoor environment using a robot and DASY3 measurement system with the following probes: E-field probe ER3DV6 and $\mathrm{H}$-field probe H3DV6 (SPEAG, Zurich, Switzerland). Reflections of the environment and robot arm were minimized by placing absorbers.

Measurement setup for SAR compliance testing consisted of a robot (Staubli Type Rx90B L, Staubli, Pfäffikon, Switzerland), a DASY4 measurement system (SPEAG), a power meter (Agilent E4419B), power sensors $(8482 \mathrm{H}$, Agilent), a directional coupler (HP775D Dual Directional Coupler 450-940 MC, Agilent), an RF termination (Meca 480-1, Agilent), a

TABLE 1. Four Commercially Available Walkie-Talkies

Walkie-talkie (manufacturer)

COBRA MT 500 (Cobra Electronics, Chicago, IL) TwinTalker 3300 (Topcom, Tilburg, The Netherlands) TwinTalker 1300 (Topcom, Tilburg, The Netherlands) Alecto FR-20 (Alecto Electronics, 's-Hertogenbosch, The Netherlands) generator (HP8647A, Agilent), a network analyzer (HP8753E, Agilent), and the flat phantom (ELI4, SPEAG) filled with head simulating liquid (HSL450, SPEAG). The DASY4 measurement system consisted of data acquisition electronics (DAE3 from SPEAG) and a dosimetric probe (ET3DV6 from SPEAG). Figure 2 shows setup for performing dosimetric measurements. The dosimetric system (probe and data acquisition electronics) was attached to a robot. Robot and data acquisition electronics were connected to a server and managed through the graphical user interface of a laptop or desktop computer. Walkie-talkies and the generic walkie-talkie were placed at short

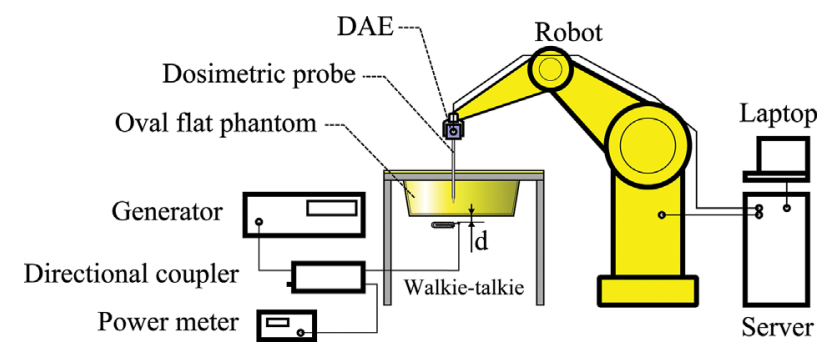

Fig. 2. Drawing of setup for dosimetric measurements. 
distance (d) below oval flat phantom. Oval flat phantom was filled with head simulating liquid (HSL450 from SPEAG) as suggested by IEC 62209 [IEC, 2001]. Dielectric properties of the liquid at $450 \mathrm{MHz}$ were $\sigma=0.87 \mathrm{~S} / \mathrm{m}$ and $\varepsilon_{r}=43.5$. Separations between liquid and walkie-talkie were $2 \mathrm{~mm}$ (touch position), 15, 30, and $50 \mathrm{~mm}$. For the generic walkie-talkie we did not measure in touch position (we did not want the ground plane of the generic walkie-talkie to make contact with the shell of the phantom) and at $50 \mathrm{~mm}$ (due to antenna's low efficiency, we could not measure at this distance). In the case of the generic walkie-talkie, it was connected to a radiofrequency (RF) generator. Input power of antennas was measured by a power meter via a directional coupler.

The worst-case uncertainty of the DASY4 measurement system was mentioned in the manual [SPEAG, 2008] provided with the system. Combined standard uncertainty on the local-averaged SAR in $1 \mathrm{~g}$ and $10 \mathrm{~g}$ equals $10.9 \%$ and $10.7 \%$, respectively; expanded standard uncertainty on local-averaged SAR in $1 \mathrm{~g}$ and $10 \mathrm{~g}$ equals $21.9 \%$ and $21.4 \%$, respectively. This worst-case uncertainty budget for DASY4 was assessed according to IEEE 1528 [IEEE, 2003]. For specific tests and configurations, uncertainty can be considerably smaller [SPEAG, 2008].

The commercially available walkie-talkies were placed below the flat phantom with their push-to-talk button fixed during measurement in order to transmit continuously. Before every measurement, fullycharged batteries were placed in walkie-talkies to ensure transmission at maximum power. A walkietalkie uses half-duplex communication resulting in a varying duty cycle from $0 \%$ to $100 \%$. A continuously transmitting device has a duty-cycle of $100 \%$. Hence, a worst-case duty cycle of $100 \%$ was selected for compliance testing.

Besides four commercially available walkietalkies, we also assessed peak local-averaged SAR in $1 \mathrm{~g}$ and $10 \mathrm{~g}$ for the hand-made generic walkie-talkie. Measured peak local-averaged SAR values were compared with simulations.

\section{Numerical Methods}

We employed the finite-difference time-domain (FDTD) solver available in the three-dimensional fullwave electromagnetic software package SEMCAD-X (SPEAG) for performing numerical analysis in free space and below the oval flat phantom. In FDTD calculations, simulation domain is finite and boundary conditions are used to mimic free space. We applied uni-axial perfectly matched layers (UPML) at the boundaries. The number of layers was automatically set by the FDTD solver to obtain a selected efficiency of $99.9 \%$. Padding (minimum distance between absorbing boundaries [UPML] and the bounding box around the walkie-talkie or combination of walkietalkie and the flat phantom) was a quarter of a wavelength. Grid step for the helical antenna equaled $0.5 \mathrm{~mm}$ (133 times smaller than a tenth of the wavelength in free space at $450 \mathrm{MHz}$ ), and maximum grid step in the flat phantom was $2 \mathrm{~mm}$ (5 times smaller than a tenth of the wavelength in the tissue simulating liquid at $450 \mathrm{MHz}$ ). Based on these settings, we estimated uncertainty on peak localaveraged SAR from the study of Bakker et al. [2010, 2011]. Expanded uncertainty $U(k=2)$ on $\mathrm{SAR}_{10 \mathrm{~g}}$ in the flat phantom filled with tissue-simulating liquid equaled $11.9 \%$ (there is no uncertainty on dielectric properties of tissue-simulating liquid because the values are specified by standards).

\section{RESULTS}

\section{Free-Space Evaluation of the Generic Walkie-Talkie}

Figure 3 shows dimensions of the derived generic walkie-talkie operating at $446 \mathrm{MHz}$ and the coordinate system used in this study. Model dimensions and dielectric parameters are listed in Table 2. These values can be modified to specific dimensions of different types of walkie-talkies. The helical antenna fits in a dielectric cover with a relative permittivity $\left(\varepsilon_{r}\right)$ of 3.9 and a conductivity $(\sigma)$ of $12 \mathrm{mS} / \mathrm{m}$.

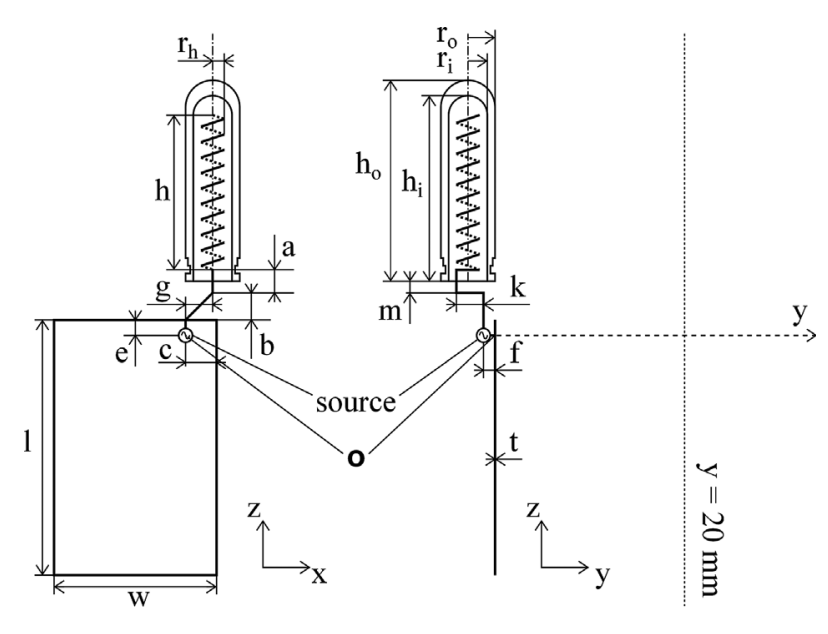

(a)

(b)

Fig. 3. Generic walkie-talkie in (a) xz-plane and (b) yz-plane. Measurement line $(y$-axis) and plane $(y=20 \mathrm{~mm})$ for near fields are also shown. 
TABLE 2. Dimensions of a Walkie-Talkie Model

\begin{tabular}{llcc}
\hline Helical antenna & & & Ground plane \\
\hline$a$ & $4.2 \mathrm{~mm}$ & $l$ & $80 \mathrm{~mm}$ \\
$b$ & $9.8 \mathrm{~mm}$ & $w$ & $45 \mathrm{~mm}$ \\
$c$ & $10 \mathrm{~mm}$ & $t$ & $0.5 \mathrm{~mm}$ \\
$e$ & $5 \mathrm{~mm}$ & & Antenna housing \\
\cline { 2 - 4 }$f$ & $2 \mathrm{~mm}$ & $r_{i}$ & $3.5 \mathrm{~mm}$ \\
$g$ & $8.54 \mathrm{~mm}$ & $r_{o}$ & $7 \mathrm{~mm}$ \\
$h$ & $42 \mathrm{~mm}$ & $\varepsilon_{r}$ & 3.9 \\
$k$ & $7.2 \mathrm{~mm}$ & $\sigma$ & $12 \mathrm{mS} / \mathrm{m}$ \\
$n_{\text {turns }}$ & 21 & $h_{i}$ & $51 \mathrm{~mm}$ \\
$r_{h}$ & $2.4 \mathrm{~mm}$ & $h_{o}$ & $53 \mathrm{~mm}$ \\
$r_{w}$ & $0.5 \mathrm{~mm}$ & $m$ & $1 \mathrm{~mm}$ \\
\hline
\end{tabular}

We realized a balanced feed current at terminals of the measurement model with a bazooka or a sleeve balun [Balanis, 1982]. Operating frequency of the bazooka balun was $446 \mathrm{MHz}$. (The bazooka balun is not shown in Fig. 1b).

Return loss $\left|\mathrm{S}_{11}\right|$ in $\mathrm{dB}$ of the model with respect to $50 \Omega$ is shown in Figure 4. A very good agreement is observed between simulations and measurements. Resonance frequency $f_{\text {res }}$ calculated by the FDTD tool is $436.5 \mathrm{MHz}$ and deviates only $0.3 \%$ of the measured value $f_{\text {res }}$, that is, $435.2 \mathrm{MHz}$. Simulated input impedance at resonance $Z_{\text {in,res }}$ equaled $20.8 \Omega$, measured input impedance equaled $24.5 \Omega$. At operating frequency of $446 \mathrm{MHz}$, input impedance of model $Z_{\text {in }}$ was $28.6+\mathrm{i} 30.4 \Omega$ and $23.1+\mathrm{i} 29 \Omega$ for measurement and FDTD simulation, respectively.

We already mentioned that dielectric parameters of the cover (relative permittivity of 3.9 , conductivity of $12 \mathrm{mS} / \mathrm{m}$.) around the antenna were derived by tuning these parameters until simulations agreed with measurements in term of resonance frequency, return loss, and near fields.

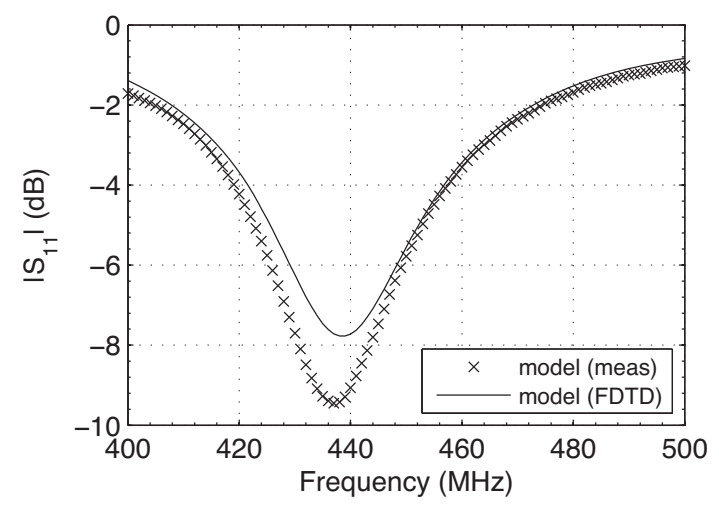

Fig. 4. Return loss $\left|\mathrm{S}_{11}\right|$ of model.
Radiation efficiency $(\eta)$ of an antenna is defined as ratio of radiated power $\left(P_{\text {rad }}\right)$ and input antenna power $\left(P_{\text {in }}\right)$, or $\eta=P_{\text {rad }} / P_{\text {in }}$ [Balanis, 1982]. Radiation efficiency was calculated using FDTD simulations. For the generic walkie-talkie, we obtained an efficiency of $53 \%$ at $446 \mathrm{MHz}$. This low efficiency was due to the reflection at input terminals of the helical antenna and ohmic losses in housing around the helical antenna introduced by the conductivity $(\sigma)$ of $12 \mathrm{mS} / \mathrm{m}$.

Far-field behavior of the generic walkie-talkie has been investigated numerically in terms of gain $(G)$. Using FDTD, a gain of 1.07 for simulation model was obtained. This value is lower than the standard gain of a half-wave dipole antenna $\left(G_{d}\right)$, mainly due to losses in the dielectric cover around the helical antenna of the model. ERP can be calculated as follows [FCC, 1997]:

$$
\mathrm{ERP}=\frac{G}{G_{d}} P_{\text {in }}
$$

Effective radiated power was measured for the generic walkie-talkie with coating. For an input power of $10 \mathrm{~mW}$, measured maximum ERP was $4.9 \mathrm{~mW}$ (measurement uncertainty was $3.3 \mathrm{~dB}$ ). This agreed well with simulated ERP of $4.8 \mathrm{~mW}$ (relative error was $2 \%$ ). Based on simulated ERP for an input power of $10 \mathrm{~mW}$, we calculated that input power of the helical antenna (or output power of generator) must be set to $1042 \mathrm{~mW}$ to obtain maximum allowed ERP of $500 \mathrm{~mW}$ as specified by the PMR 446 [ERC, 1998] and FRS standard [FCC, 2015]. This input power is used below to determine compliance of the model with safety guidelines [ICNIRP, 1998; FCC, 2001].

Near fields of the model were simulated and measured in the $y$-plane at $20 \mathrm{~mm}$ from the model $(y=20 \mathrm{~mm})$ according to the setup shown in Figure 3. This plane was parallel to the ground plane of the generic walkie-talkie (or PCB of real devices) at a distance that might be considered a typical separation distance value when a walkie-talkie is operated in front of the face. ERP was set to $500 \mathrm{~mW}$. Figure 5 shows simulated ([a] and [b]) and measured ([c] and [d]) RMS electric $E_{\mathrm{rms}}$ ([a] and [c]) and RMS magnetic $H_{\text {rms }}([\mathrm{b}]$ and [d]) near fields of the model at $y=20 \mathrm{~mm}$. One observes that distribution of the electric and magnetic field as well as absolute field values of the simulations agree well with measurements. Dots in Figure 5 show the position of the maximum field values. The position of the maximum RMS E-field and $\mathrm{H}$-field was situated near the helical antenna and terminals of the model, respectively. In the plane $y=20 \mathrm{~mm}$, simulated $E_{\mathrm{rms}, \max }$ (Fig. 5a) 


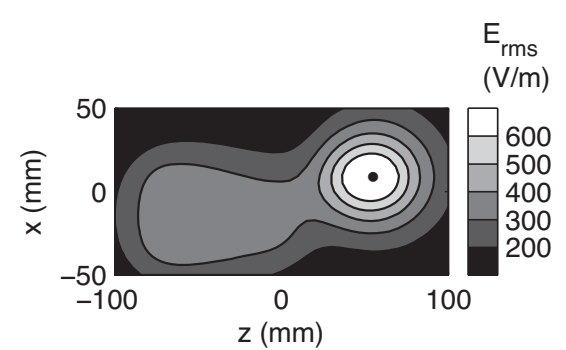

(a)

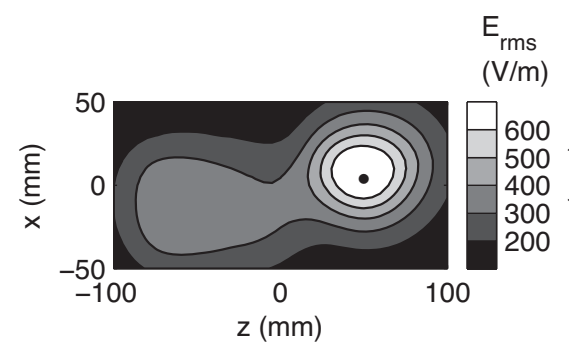

(c)

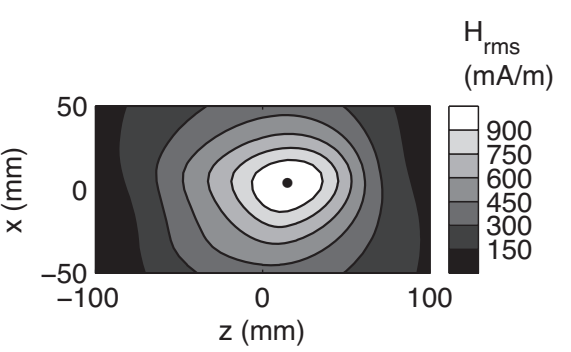

(b)

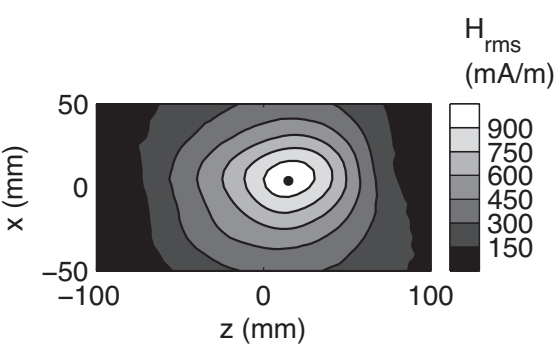

(d)

Fig. 5. (a) and (b) simulated and (c) and (d) measured electromagnetic near fields at $y=$ $20 \mathrm{~mm}$ of generic walkie-talkie.

equals $762 \mathrm{~V} / \mathrm{m}$ in the point $(x=10 \mathrm{~mm}, z=55 \mathrm{~mm})$ and deviates only $1.6 \%$ from measured field value, that is, $E_{\mathrm{rms}, \max }$ of $750 \mathrm{~V} / \mathrm{m}$ in point $(x=5 \mathrm{~mm}$, $z=50 \mathrm{~mm}$ ). In the same plane $y=20 \mathrm{~mm}$, simulated $H_{\mathrm{rms}, \max }$ is $1080 \mathrm{~mA} / \mathrm{m}$ in $(x=5 \mathrm{~mm}, z=15 \mathrm{~mm})$ and deviates $7.7 \%$ from measured $H_{\mathrm{rms} \text {,max }}$ of $1003 \mathrm{~mA} / \mathrm{m}$ in $(x=5 \mathrm{~mm}, z=15 \mathrm{~mm})$.

\section{Free-Space Evaluation of Four Commercially Available Walkie-Talkies}

Besides the COBRA MT500, three other PMR 446 radios were acquired. In this section, the radiation characteristics of all the walkie-talkies (Table 1) and near-field behavior of the COBRA MT500 are investigated.

Radiation characteristics of real devices were measured at SP Technical Research Institute of Sweden (Borås, Sweden). Table 3 lists TRP, maximum ERP, and averaged ERP. Maximum effective

TABLE 3. Total Radiated Power and Effective Radiated Power of Four Commercial Walkie-Talkies

\begin{tabular}{lccc}
\hline Walkie-talkie & $\begin{array}{c}\text { TRP } \\
(\mathrm{mW})\end{array}$ & $\begin{array}{c}\text { Max. ERP } \\
(\mathrm{mW})\end{array}$ & $\begin{array}{c}\text { Avg. ERP } \\
(\mathrm{mW})\end{array}$ \\
\hline $\begin{array}{l}\text { COBRA MT500 } \\
\text { TwinTalker }\end{array}$ & 105 & 138 & 117 \\
$\quad 3300$ & 59 & 79 & 68 \\
$\begin{array}{l}\text { TwinTalker } \\
\quad 1300\end{array}$ & 51 & 69 & 63 \\
\begin{tabular}{l} 
Alecto FR-20 \\
\hline
\end{tabular} & 44 & 51 & 47 \\
\hline
\end{tabular}

radiated power varied from $51 \mathrm{~mW}$ to $138 \mathrm{~mW}$. This is about 9.8-3.6 times below specification of allowed ERP for PMR 446 radios.

Near fields of COBRA MT500 were measured and compared to the model of the walkie-talkie (which has dimensions based on dimensions of the COBRA MT500). We positioned the walkie-talkie in the same way as the model (see Fig. 3) such that the feed point coincided with the origin of the coordinate system. We normalized fields to the maximum field value in the plane $y=20 \mathrm{~mm}$, because we were not able to determine input power for the real walkietalkie. Figure 6 shows normalized electric and magnetic field in a plane for the real walkie-talkie (only measurements). These distributions agree well with simulated (see Fig. 5a and c) and the measured (see Fig. $5 b$ and d) near field distributions of the generic walkie-talkie. Compared to the real walkie-talkie, maximum relative error for the measured and simulated model on the normalized electric field were only $1.2 \%$ and $5.2 \%$, respectively. For normalized magnetic near field, maximum relative error for the measured and simulated model was $5.2 \%$ and $5.5 \%$, respectively. These low deviations show that the model behaves electromagnetically like a real walkietalkie and can be used for analysis of interaction with the human body.

\section{SAR Assessment of Real Walkie-Talkies}

Figure 7 shows peak local-averaged SAR in $1 \mathrm{~g}$ and $\left(\mathrm{SAR}_{1 \mathrm{~g}}\right)$ and $10 \mathrm{~g}\left(\mathrm{SAR}_{10 \mathrm{~g}}\right)$ of the four 


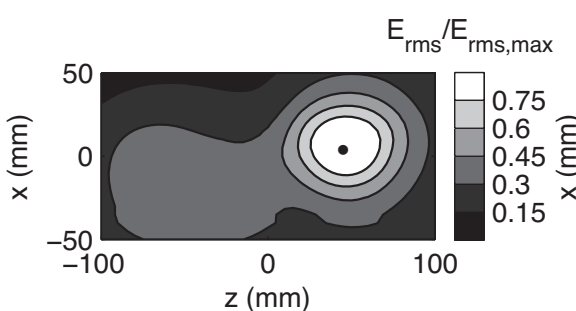

(a)

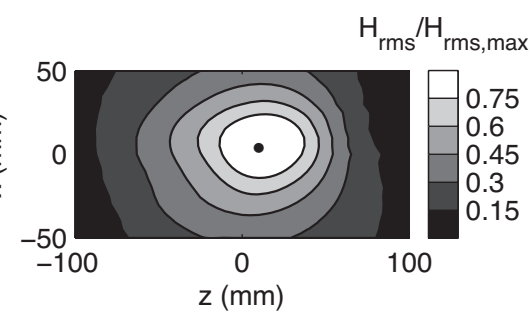

(b)

Fig. 6. Normalized RMS (a) electric and (b) magnetic near field at $y=20 \mathrm{~mm}$ of a real walkie-talkie.

commercial walkie-talkies for a duty-cycle of $100 \%$ (i.e., continuously speaking for at least $6 \mathrm{~min}$.) The Alecto was not measured at a distance of $15 \mathrm{~mm}$ between the device and the liquid inside the flat phantom. Except for the touch position, the COBRA MT500 showed a higher SAR in $1 \mathrm{~g}$ and $10 \mathrm{~g}$ than the walkie-talkies from Topcom (Tilburg, The Netherlands) and Alecto ('s-Hertogenbosch, The Netherlands).

Highest values of the $\mathrm{SAR}_{1 \mathrm{~g}}$ and $\mathrm{SAR}_{10 \mathrm{~g}}$ occurred when the walkie-talkie touched the flat phantom: $\mathrm{SAR}_{1 \mathrm{~g}}=0.52 \mathrm{~W} / \mathrm{kg}$ (Alecto) and $\mathrm{SAR}_{10 \mathrm{~g}}=0.35 \mathrm{~W} / \mathrm{kg}$ (Alecto). These values for the touch position comply
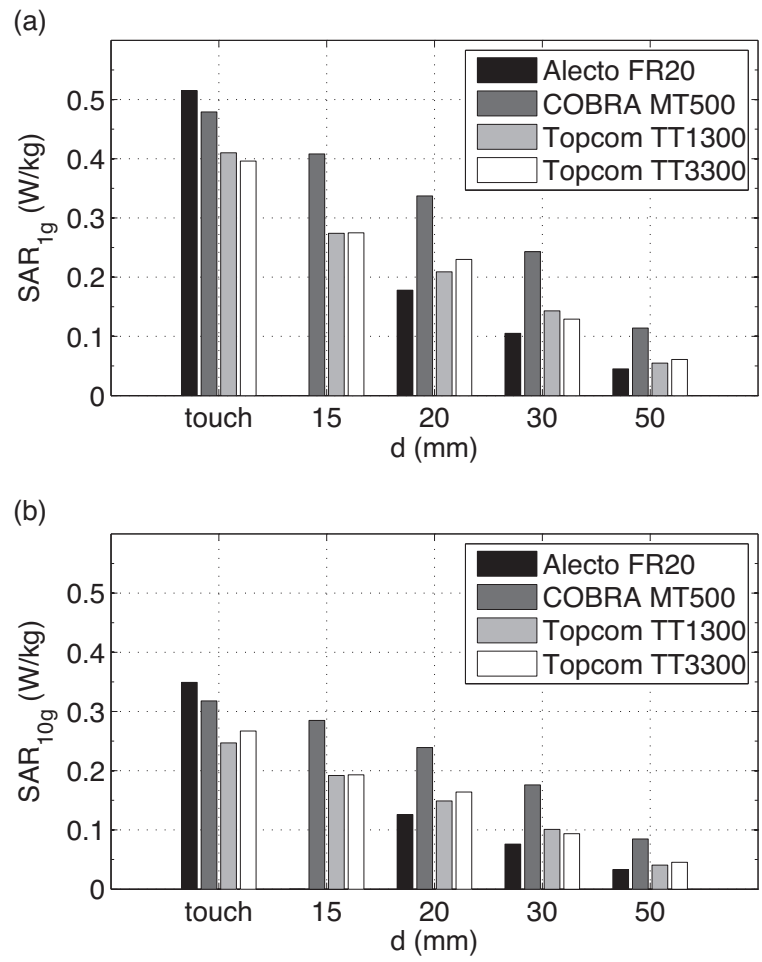

Fig. 7. Peak local-averaged SAR in (a) $1 \mathrm{~g}$ and (b) $10 \mathrm{~g}$ induced in oval flat phantom by four commercially available walkietalkies with duty cycle of $100 \%$. with the SAR-limits for $1 \mathrm{~g}$ (i.e., $1.6 \mathrm{~W} / \mathrm{kg}$ ) and $10 \mathrm{~g}$ (i.e., $2 \mathrm{~W} / \mathrm{kg}$ ), respectively, even for a worst-case duty cycle of $100 \%$.

Cecil et al. [2014] and Dimbylow et al. [2003] reported peak $10 \mathrm{~g}$ SAR values up to about $50 \%$ of ICNIRP limit for general public exposure for TETRA transmitters. This is higher than the maximum of $0.35 \mathrm{~W} / \mathrm{kg}$ observed in our study. Besides the difference in phantom (human body model vs. flat) and distance between the walkie-talkie and the phantom, the difference was mainly due to the difference in applied power: Cecil et al. as well as Dimbylow et al. used a transmitted power of $0.25 \mathrm{~W}$ for the hand-held TETRA devices whereas in our study, SAR values were for typical TRP-values (in free space) of walkie-talkies ranging from $44 \mathrm{~mW}$ to $105 \mathrm{~mW}$ (Table 3).

\section{SAR Assessment of Generic Walkie-Talkie}

The input power of the generic walkie-talkie was set to $10 \mathrm{~mW}$ during measurement taking into account reflection losses. We validated use of the generic walkie-talkie as a substitute for real devices by comparing peak local-averaged SAR of real devices with ones of the model for the same ERP of the real devices and for the same magnetic near field. Above, we discussed ERP values of the four walkie-talkie devices. We observed that ERP of considered devices was at least 3.6 times smaller than maximum allowed ERP for PMR 446 radios. We rescaled ERP of the generic walkie-talkie (measured value of $4.9 \mathrm{~mW}$ and simulated ERP value of $4.8 \mathrm{~mW}$ for an antenna input power of $10 \mathrm{~mW}$ ) to values listed in Table 3 . The antenna input power needed to reach ERP of the walkietalkies was applied during dosimetric measurement. Figure 8 shows the $\mathrm{SAR}_{1 \mathrm{~g}}$ and $\mathrm{SAR}_{10 \mathrm{~g}}$ of the generic walkie-talkie with the same ERP of the COBRA MT500. We observed that the generic walkie-talkie overestimated peak local-averaged SAR of the real devices (Fig. 7). Rescaling input 
(a)

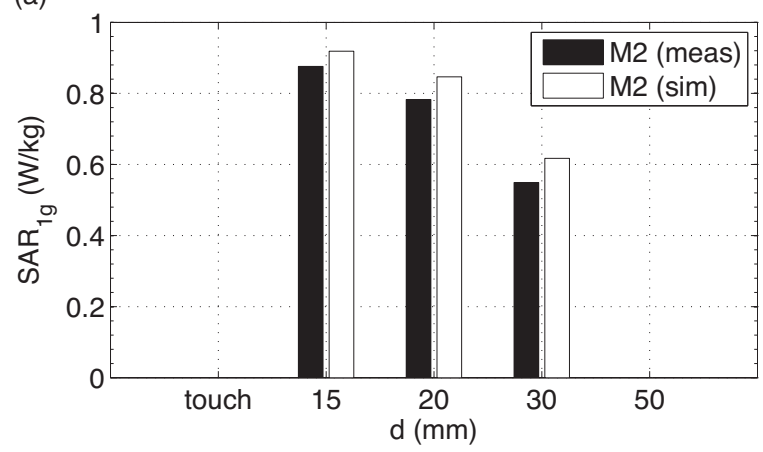

(b)

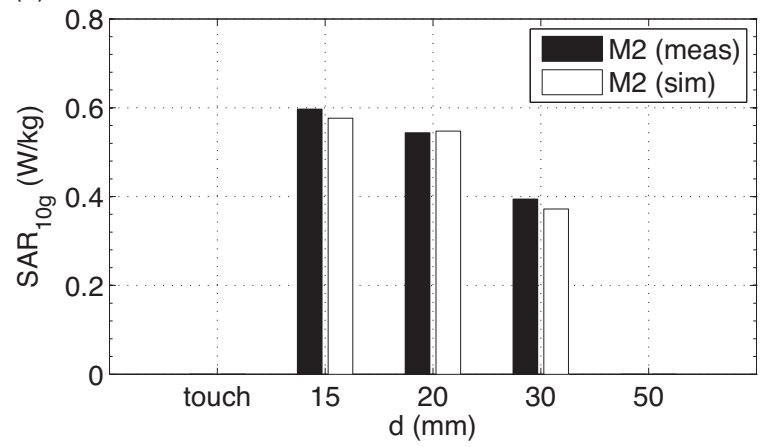

Fig. 8. Peak local-averaged SAR in (a) $1 \mathrm{~g}$ and (b) $10 \mathrm{~g}$ induced in flat phantom by generic walkie-talkie for same ERP as COBRA MT500 (100\% duty cycle).

power based on ERP (a far-field characteristic) was inadequate for assessment of SAR in human tissue despite the use of the helical antenna with coating from the COBRA MT500. The ratio for $\mathrm{SAR}_{1 \mathrm{~g}}$ and $\mathrm{SAR}_{10 \mathrm{~g}}$ (for ERP equal to ERP of the devices) of the model and device was 2.5 and 2.3, respectively. The deviation originates from uncertainties on ERP and SAR measurements, simplifications of the model with respect to the real device and from differences in coupling when the device or model operates at close distance to the phantom. Ratio for the other real walkie-talkies was maximum 2.8 (Topcom tt3300) and 2.5 (Topcom tt3300) for $\mathrm{SAR}_{1 \mathrm{~g}}$ and $\mathrm{SAR}_{10 \mathrm{~g}}$, respectively. For further numerical analysis of the $\mathrm{SAR}_{10 \mathrm{~g}}$ in the flat phantom using the generic walkie-talkie and based on a rescaling of ERP, we will take into account a correction factor of two for $\mathrm{SAR}_{10 \mathrm{~g}}$ value.

A better quantity for validating the generic walkie-talkie as a substitute for a real device for assessing peak local-averaged SAR is the magnetic near field. Kuster and Balzano [1992] showed that the SAR is mainly proportional to incident magnetic near field. We rescaled the maximum of the magnetic near field in a plane at a distance of $20 \mathrm{~mm}$ of the ground plane of the generic walkie-talkie to the maximum value of the magnetic field in the same plane above the real device. Peaklocal averaged SAR of the generic walkie-talkie for a rescaling based on magnetic field is shown in Figure 9. We observed that maximum deviation on the peak local-averaged SAR was less than $26 \%$. Thus, magnetic near field is a better quantity than far-field parameter ERP for estimating peak localaveraged SAR of a real device.

Finally, we estimated worst-case peak localaveraged SAR for the walkie-talkies. We determined peak local-averaged SAR in $1 \mathrm{~g}$ and $10 \mathrm{~g}$ for an input power that gives an ERP of $500 \mathrm{~mW}$ in free space (we assumed a duty cycle of $100 \%$ for worst-case evaluation) for the generic walkie-talkie and taking into account a correction factor of two (see above). Figure 10 shows that the generic walkie-talkie is not compliant to the FCC limit of $1.6 \mathrm{~W} / \mathrm{kg}$ in a cube of $1 \mathrm{~g}$ at $15 \mathrm{~mm}$. The worst-case peak local-averaged SAR in $1 \mathrm{~g}$ of tissue exceeded the FCC limit by up to $7 \%$ (based on simulated value). If we compared peak local-averaged SAR in $10 \mathrm{~g}$ of tissue with ICNIRP basic restriction of $2 \mathrm{~W} / \mathrm{kg}$, then we observed that the walkie-talkies are compliant.
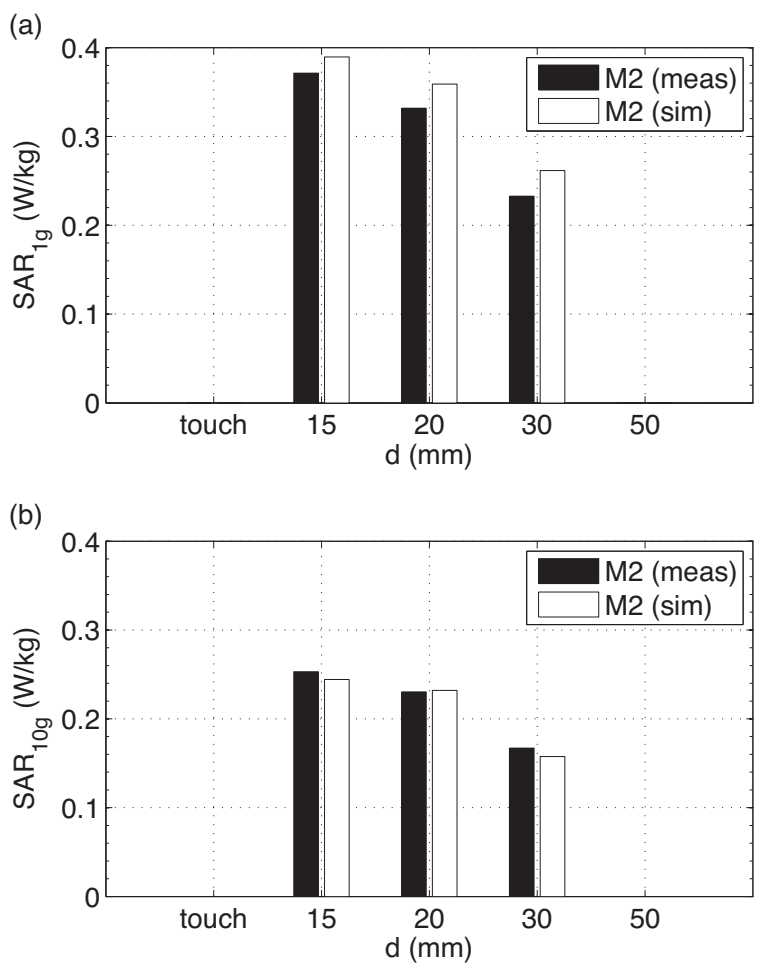

Fig. 9. Peak local-averaged SAR in (a) $1 \mathrm{~g}$ and (b) $10 \mathrm{~g}$ induced in the oval flat phantom by generic walkie-talkie for same magnetic field at $20 \mathrm{~mm}$ above ground plane as COBRA MT500 (100\% duty cycle). 


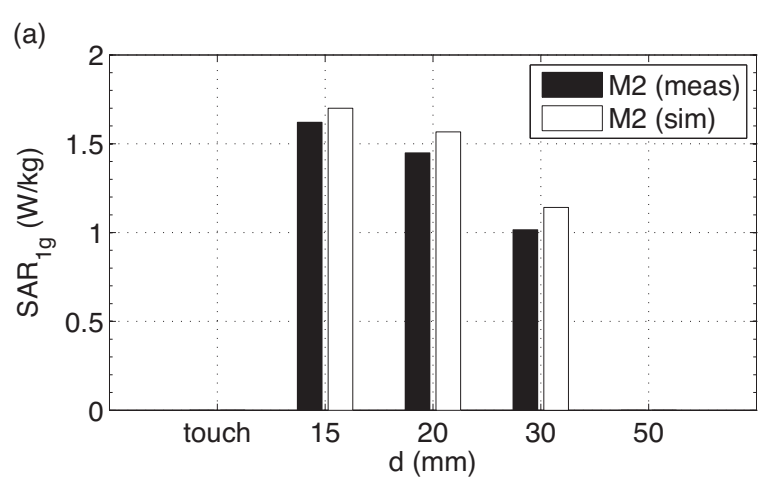

(b)

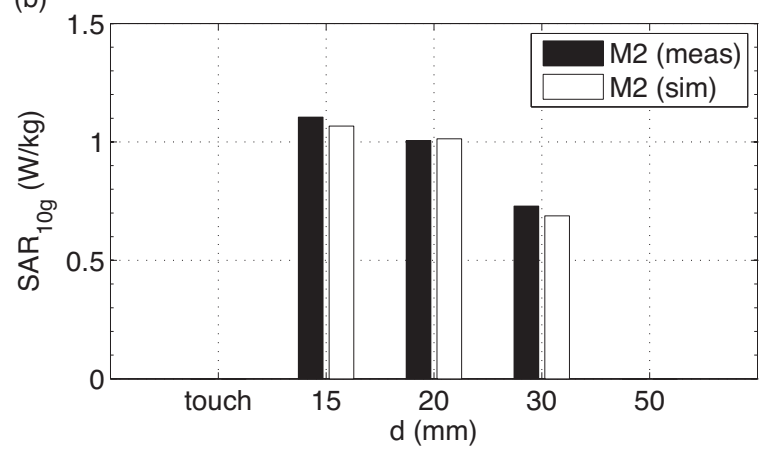

Fig. 10. Worst-case assessment of peak local-averaged SAR in (a) $1 \mathrm{~g}$ and (b) $10 \mathrm{~g}$ induced by generic walkie-talkie for $\mathrm{ERP}=500 \mathrm{~mW}(100 \%$ duty cycle $)$.

\section{CONCLUSIONS}

An accurate model for a walkie-talkie has been developed and very good agreement reported from simulations and measurements in terms of reflection and transmission characteristics. Near fields of the model have been validated with measured near fields of a real walkie-talkie. A relative error of less than $5.5 \%$ has been observed on the near electric and magnetic field indicating that the walkie-talkie model behaves electromagnetically as a real walkie-talkie. Therefore, the model can be used to determine electromagnetic interaction with the human body and to test compliance with safety limits. Total radiated power and effective radiated power were measured for four real or commercially available walkie-talkies and a generic walkie-talkie. Measured effective radiated power of the real walkie-talkies is about 3.6-9.8 times lower than allowed effective radiated power for PMR 446 radios.

We found that rescaling the input power of the generic walkie-talkie based on the ERP (a far-field characteristic) is inadequate for assessment of SAR in human tissue: we observed a ratio for $\mathrm{SAR}_{1 \mathrm{~g}}$ and $\mathrm{SAR}_{10 \mathrm{~g}}$ between the model and the device of 2.5 and 2.3. A better quantity for validating the generic walkie-talkie as a substitute for a real device for assessing peak local-averaged SAR is the magnetic near field with deviations of less than $26 \%$.

We investigated compliance to SAR safety limits of a walkie-talkie. An effective radiated power as specified by the PMR 446 radio standard can cause a local-averaged SAR, which exceeds limits. However, based on effective radiated power of four real walkietalkies, we showed that peak local-averaged SAR values are unlikely to exceed current SAR limits.

\section{REFERENCES}

Bakker JF, Paulides MM, Christ A, Kuster N, van Rhoon GC. 2010. Assessment of induced SAR in children exposed to electromagnetic plane waves between $10 \mathrm{MHz}$ and 5.6 GHz. Phys Med Biol 55:3115-3130.

Bakker JF, Paulides MM, Christ A, Kuster N, van Rhoon GC. 2011. Corrigendum for assessment of induced SAR in children exposed to electromagnetic plane waves between $10 \mathrm{MHz}$ and 5.6 GHz. Phys Med Biol 56:2883.

Balanis CA. 1982. Antenna theory, analysis and design. Hoboken, NJ: John Wiley\& Sons. pp 58, 368.

Balzano Q, Garay O, Manning T. 1995. Electromagnetic energy exposure of the users of portable cellular telephones. IEEE Trans Vehic Technol 44:390-403.

Cecil S, Neubauer G, Rauscha F, Stix G, Müller W, Breithuber Glanzer M. 2014. Possible risks due to exposure of workers and patients with implants by TETRA transmitters. Bioelectromagnetics 35:192-200.

Dimbylow P, Khalid M, Mann S. 2003. Assessment of specific energy absorption rate (SAR) in the head from a TETRA handset. Phys Med Biol 48:3911-3926.

European Radio Communications Committee (ERC). 1998. ERC decision of 23 November 1998 on the harmonised frequency band to be designated for PMR 446 (ERC/DEC/ (98) 25), Brussels, Belgium.

European Telecommunications Standards Institute (ETSI). 2001a. ETSI European standard, electromagnetic compatibility and radio spectrum matters (ERM); land mobile service; radio equipment using integral antennas intended primarily for analogue speech; part 1: Technical characteristics and methods of measurement. ETSI EN 300 296-1, SophiaAntipolis, France.

European Telecommunications Standards Institute (ETSI). 2001b. ETSI European standard, electromagnetic compatibility and radio spectrum matters (ERM); land mobile service; radio equipment using integral antennas intended primarily for analogue speech; part 2: Harmonized EN covering essential requirements under article 3.2 of the R\&TTE directive. ETSI EN 300 296-2, Sophia-Antipolis, France.

Federal Communications Commission (FCC). 1997. Evaluating compliance with FCC guidelines for human exposure to radiofrequency electromagnetic fields. Tech. Rep. OET bulletin 65, Washington, DC.

Federal Communications Commission (FCC). 2001. Evaluating compliance with FCC guidelines for human exposure to radiofrequency electromagnetic fields. Tech Rep Supplement $\mathrm{C}$ to bulletin 65, Washington, DC.

Federal Communications Commission (FCC). Undated. Family radio service (FRS). Available from: http://www.fcc.gov/ encyclopedia/family-radio-service-frs [Last accessed 11 Aug 2015], Washington, DC. 
Institute of Electrical and Electronics Engineers (IEEE). 2003. IEEE recommended practice for determining the spatialpeak specific absorption rate (SAR) in the human body from wireless communications devices: Experimental techniques. Std. 1528-2003, New York, NY.

International Commission on Non-Ionizing Radiation Protection (ICNIRP). 1998. Guidelines for limiting exposure to timevarying electric, magnetic, and electromagnetic fields. Health Phys 74:494-522.

International Electrotechnical Commission (IEC). 2001. Procedure to measure the specific absorption rate (SAR) for hand-held mobile wireless devices in the frequency range of $300 \mathrm{MHz}$ to $3 \mathrm{GHz}$. IEC62209, Geneva, Switzerland.

International Electrotechnical Commission (IEC). 2005. Human exposure to radio frequency fields from hand-held and body-mounted wireless communication devices-human models, instrumentation, and procedures-part 2: Procedure to determine the specific absorption rate (SAR) in the head and body for $30 \mathrm{MHz}$ to $6 \mathrm{GHz}$ handheld and bodymounted devices used in close proximity to the body. TC/ SC106/90/NP, Geneva, Switzerland.
Koulouridis S, Nikita KS. 2004. Study of the coupling between human head and cellular phone helical antennas. IEEE Trans Electromagn Compat 46:62-70.

Kuster N, Balzano Q. 1992. Energy absorption mechanism by biological bodies in the near field of dipole antennas above 300MHz. IEEE Trans Veh Technol 41:17-23.

Kuster N, Balzano Q, Lin JC. 1997a. Mobile communications safety. London, UK: Chapman \& Hall. pp 21-22.

Kuster N, Kastle R, Schmid T. 1997b. Dosimetric evaluation of hand-held mobile communications equipment with known precision. IEICE Trans Commun E80-B:645-652.

Lazzi G, Gandhi O. 1998. On modeling and personal dosimetry of cellular telephone helical antennas with the FDTD code. IEEE Trans Antennas Propag 46:525-530.

Meyer F, Palmer K, Jakobus U. 2001. Investigation into the accuracy, efficiency and applicability of the method of moments as numerical dosimetry tool for the head and hand of a mobile phone user. Appl Comput Electromagn Soc J 16:114-125.

SPEAG. 2008. DASY4 System Handbook. Zurich, Switzerland.

TCO Development. 2008. TCO'01 Certification of Mobile Phones. Stockholm, Sweden. 